\title{
Instrumental responding to internal cues associated with REM sleep
}

J. SALAMY, ${ }^{2}$ Langley Porter Neuropsychiatric Institute, 401 Parnassus Ave., San Francisco, Calif. 94122

This paper examined sleeping Ss' ability to discriminate and respond to internal cues associated with REM sleep. Ss were instructed to press a switch, taped to their hands, four times as soon as they started dreaming. Improvement in performance was obtained when $S s$ were placed on an avoidance paradigm such that response failure resulted in awakening. $A$ preliminary attempt was made to formulate the findings in terms of a motivational context. It was concluded that sleeping $S s$ can perform an instrumental response to cues associated with REM sleep.

There is considerable evidence demonstrating that humans are capable of making specified responses during sleep (Oswald, Taylor, \& Treisman, 1960; Granda \& Hammack, 1961; Williams, Morlock, \& Morlock, 1963, 1966; Antrobus, Antrobus, \& Fisher, 1965). In an experiment investigating the effects of reinforcement on behavioral responding during sleep, Williams et al $(1963,1966)$ instructed Ss to close a switch (which was taped to their hands) whenever a tone was presented. After 3 nights on this instruction condition, contingent punishment was introduced. Failure to respond within $4 \mathrm{sec}$ of the tone resulted in the following sequence of events: A high-intensity fire alarm was sounded, followed by flashing lights and shocks to the leg. While all Ss displayed the capacity to respond, the strongest reinforcement effect occurred in stage REM, where the proportion of correct responses went from $10 \%$ under the instructions condition to $50 \%$ on the avoidance nights. Many of these responses occurred without EEG evidence of prior awakening.

When the average proportion of

Table 1

Number of Correct Responses for Each $S$ for Each Night

\begin{tabular}{lrrrrrr}
\hline & \multicolumn{5}{c}{ Nights } & \\
\cline { 2 - 6 } Subjects & 1 & 2 & 3 & 4 & 5 & Total \\
\hline EM & 0 & 2 & 6 & 1 & 3 & 12 \\
JG & 0 & 0 & 11 & 2 & 3 & 16 \\
MP & 1 & 3 & 7 & 1 & 0 & 12 \\
JH & 1 & 2 & 3 & 3 & 3 & 12 \\
DR & 2 & 1 & 1 & 3 & 2 & 9 \\
Total & 4 & 8 & 28 & 10 & 11 & 61 \\
\hline
\end{tabular}

responses in each stage was plotted for
alpha and nonalpha events, the effect of reinforcement was found to be stronger for nonalpha events (Williams et al, 1966). That is, correct responding was better when the alpha rhythm was not present.

In an experiment by Antrobus et al (1965), Ss were instructed to press a switch five times when they were asleep and dreaming and twice when asleep and not dreaming, in a counterbalanced design. Thus, unlike the previously mentioned experiments, Antrobus et al employed neither external stimulus nor reinforcement. It was found that Ss could respond with both $D$ (dream) and ND (nondream) signals.

The present research was intended to provide further information regarding sleeping Ss' ability to discriminate and respond to internal signals associated with REM sleep. The experiment combined the procedures of Antrobus et al (1965) and Williams et al (1963) in that no external stimulus was used, but response failure was negatively reinforced by awakening.

SUBJECTS AND PROCEDURE

Five male Ss each spent 6 consecutive nights in the laboratory. Night 1 was for adaptation purposes. On Night 2 , Ss received instructions to squeeze a microswitch, which was taped to their preferred hands, four times as soon as they started dreaming. On the next 3 nights (Nights 3, 4, and 5), Ss were awakened for a period of $4 \mathrm{~min}$ for failure to respond within the initial $3 \mathrm{~min}$ of any REM period (REMP). The punishments were achieved by calling the Ss' names over an intercom. Night 6 was an extinction night, on which instructions were given as on previous nights but with no awakening for response failure.

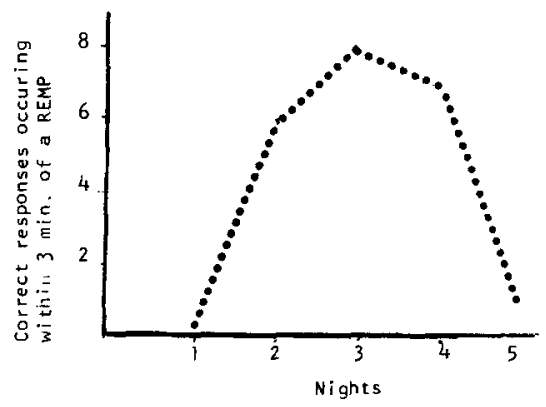

Fig. 1. Amount of correct responses occurring within the first $3 \mathrm{~min}$ of a REM period.
EEG recordings were obtained from placements located at $C_{3}-A_{2}$ according to the international 10-20 system. Eye movements were recorded from placements on the outer canthi of each eye. EMG recordings were obtained from submental placements.

\section{RESULTS}

The Ss gave a total of 61 correct REM responses over the 5 experimental nights (see Table 1). Of these, 22 , or $36 \%$, occurred within $3 \mathrm{~min}$ of REM on set.

Figure 1 shows the amount of correct responses occurring within the first $3 \mathrm{~min}$ of a REMP. Of 15 correct responses on the nonreinforcement nights, only one correct response (quadruple press) occurred within 3 min of a REMP, while of the 46 correct responses occurring on the avoidance nights, 21 , or $46 \%$, occurred within 3 min of REM onset.

The distribution of presses for all Ss over the experimental nights is shown in Fig. 2.

The difference in performance from night to night was determined by a statistic developed by Cochran (1954). This statistic tests for an abrupt change in the mean of a distribution occurring after the first N1 observations. It is distributed as chi square, with $1 \mathrm{df}$. The data from night to night were treated as an ongoing series of events. The trials in this case were REMPs or attempts to enter the REM state. N1 refers to the REMPs on a given night. Thus, the performance (number of correct responses) for all Ss on Night 1 was compared to the performance (for all $\mathrm{Ss}$ ) on Night 2, and performance on Night 2 was compared to that of Night 3 , etc. Table 2 summarizes the results of this analysis.

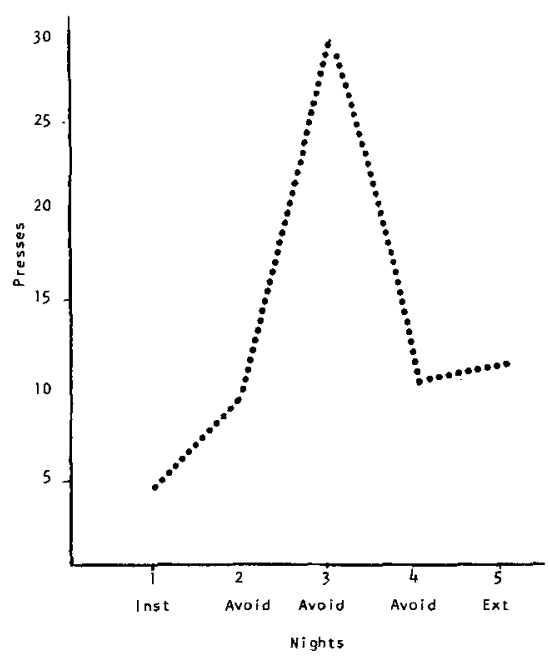

Fig. 2. The distribution of correct responses for all Ss over the experimental nights. 
Table 2

Comparison of Performance of All Ss on Each Night With That of the Succeeding Night and the Instruction Night Compared to the Extinction Night

\begin{tabular}{lll} 
Instruction & Compared to Avoidance 1 & $\chi_{2}^{2}=.66$ \\
Avoidance 1 & Compared to Avoidance 2 & $\chi_{2}^{2}=12.08^{\mathrm{a}}$ \\
Avoidance 2 & Compared to Avoidance 3 & $\chi_{2}^{2}=9.91^{\mathrm{b}}$ \\
Avoidance 3 & Compared to Extinction & $\chi_{2}^{2}=2.05$ \\
Instruction & Compared to Extinction & $\chi^{2}=4.07^{\mathrm{c}}$ \\
\hline
\end{tabular}

$a \chi^{2} \geqslant 10.83,1 \mathrm{df}, p<.001 ;{ }^{b} \chi^{2} \geqslant 6.64,1 \mathrm{df}, p<.01 ; c \cdot \chi^{2} \geqslant 3.84,1 \mathrm{df}, p<.05$

\section{DISCUSSION}

The results of this experiment are consistent with the findings of other investigators. A few responses were obtained under instructions alone, i.e., without external cues or reinforcement, as was found by Antrobus et al (1965). However, contingent reinforcement resulted in marked improvement in performance, as was found by Williams et al (1963). The present study differed from that of Williams et al in that no tones were presented, and the punishment was merely an interruption of the state; no shock or threat of it was used. Thus, performance of the avoidance response permitted the REM state to continue. The following interpretation is suggested as a possible means of accounting for the data.

The greatest amount of responding occurred on the second avoidance night (Night 3). This effect was felt to be due to a substantial degree of REM deprivation produced on the first avoidance night and continued on the second. This REM deprivation resulted from awakenings for response failure. Possibly, this increased a drive for REM sleep and made internal signals associated with REM sleep more salient. This allowed REM sleep after a correct response to serve as an adequate reinforcer. That is, the Ss were motivated to perform the avoidance response in order to obtain REM sleep and curtail further deprivation. The precipitous decline in performance on Night 4 (Avoidance Night 3) can be interpreted as due to satisfaction of the drive, i.e., a partial-satiation effect, resulting from increased responding and, therefore, increased REM time on the previous night.

In only one case did an $\mathrm{S}$ provide a correct response on Night 4 in the early part of the night before several awakenings occurred. While the effect of the avoidance treatment did promote improved performance during the extinction night compared to that of the instruction night, only one response occurred within the first $3 \mathrm{~min}$ of a REMP.

The motivational interpretation stated above is highly speculative, and the data do not warrant a conclusion with respect to it. However, the findings indicate that sleeping Ss can discriminate and respond to internal cues associated with the ongoing REM state.

\section{REFERENCES}

ANTROBUS, J. S., ANTROBUS, J., \& FISHER, C. Discrimination of dreaming and nondreaming sleep. Archives of General Psychiatry, 1965, 12, 395-401.

COCHRAN, W. G. Some methods for strengthening the common $\chi^{2}$ tests. Biometrics, 1954, 10, 417-451.

GRANDA, A. M., \& HAMMACK, J. T. Operant behavior during sleep. Science, 1961, 133, 1485-1486.

OSWALD, I., TAYLOR, A. M., \& TREISMAN, M. Discriminative responses to stimulation during human sleep. Brain, 1960, 83, 440-453.

WILLIAMS, H. L., MORLOCK, H. C., \& MORLOCK, J. V. Discriminative responses to auditory signals during sleep. Paper presented at APA convention, Philadelphia, August 31 , 1963.

WILLIAMS, H. L., MORLOCK, H. C., \& MORLOCK, J. V. Instrumental behavior during sleep. Psychophysiology, 1966, 2, 208. NOTES

1. Manuscript preparation was supported by Mental Health Training Grant No. 5-TI-MH-7082 to the University of California San Francisco Medical Center.

2. Presently, an ITP fellow at the Langley Porter Neuropsychiatric Institute, University of California San Francisco Medical Center.

\section{Vibrotactile code learning by young adolescent}

$$
\text { girls }
$$

D. D. DIESPECKER, The University of Newcastle, Newcastle, N.S.W., Australia

Three groups of young adolescent girls were trained to learn a nine-element vibrotactile code (one vibrator, three intensities, three durations of signal). Two of the groups consisted of deaf Ss, and the third group of girls had normal hearing. There was no significant difference between the groups. Although there was a significant learning effect over 10 trials, the obtained Fratio only just reached significance. Most of the errors made on the last trial were combined errors of intensity-duration.

Recent research has indicated that blind adults can learn 26-element vibrotactile codes with considerable accuracy, and also that young children are capable of learning a simple six-element vibrotactile code (Diespecker, 1967; Furner \& Diespecker, 1969). Vibrotactile learning experiments of this type with handicapped children do not appear to have been reported in the literature. The present investigation was made in an attempt to determine if young adolescent girls might be capable of learning a simple nine-element vibrotactile code.

Experimental studies of nine-element code learning have been made with university students of both sexes. In studying the effect of noise on the learning of such a code, Diespecker \& Davenport (1967) found that all 40 Ss were able to learn the code to criterion, i.e., one correct training trial. In a more recent experiment (Diespecker, in press), it was found that four groups of Ss (university students) were able to learn a nine-element code, although the durations of signals were varied over groups to determine whether or not at least one of the groups would be unable to learn the code due to the brevity of signals. There were no significant differences between these groups, but a highly significant learning effect was obtained.

In the present study, it was hypothesized that deaf girls would learn a nine-element vibrotactile code significantly better than would girls with normal hearing. This prediction was made since it seemed probable that deaf Ss would not have to contend with either sensory or physical noise and that they would therefore be able to pay more attention to such novel stimuli than would girls having normal hearing.

\section{SUBJECTS}

The Ss were two groups each of eight girls from the School for Deaf Girls at Waratah, N.S.W., and one group (eight) of girls with normal hearing. The third group of Ss attended schools in the Newcastle, N.S.W., district. Commonwealth Acoustics Laboratory average hearing loss (dB) measurements for each deaf girl were made available to $\mathrm{E}$. The values (American Standards Association) ranged from 54 to $114+$, i.e., some of the children were profoundly deaf. Although very few IQ values were available, most of the deaf girls were judged to be of average intelligence, and one of the groups (Group A) was rated by teachers to be the most proficient class in the school. Group B Ss were rated as slow learners and were slightly older. The 16 deaf girls all wore hearing aids and were competent lip-readers. The mean ages of the deaf girls were: Group A, 12.26 $(S D=1.06)$ and Group B, 14.11 